\title{
Crystal Growth Control of Functional Oxide Thin Films on Nanopatterned Substrate Surfaces
}

\author{
Yasuyuki AKITA $^{* 1}$, Yuki SUGIMOTO ${ }^{* 1}$, Keisuke KOBAYASHI $^{* 2}$, Toshimasa SUZUKI $^{* 2}$, Hideo OI $^{* 3}$, Masahiro MITA ${ }^{* 3}$ \\ and Mamoru YOSHIMOTO ${ }^{* 2, * 4}$ \\ ${ }^{* 1}$ Innovative \& Engineered Materials, Tokyo Institute of Technology, 4259-J2-46, Nagatsuta, Yoko- \\ hama, 226-8503, Japan \\ E-mail: Akita.y.aa@m.titech.ac.jp \\ *2 Taiyo Yuden Co.Ltd., 5607-2, Nakamurota, Takasaki, 370-3347, Japan \\ *3 Kyodo International Inc., 8-5-1, Chiyogaoka, Kawasaki, 215-0005, Japan \\ ${ }^{* 4}$ Patent attorney, Tokyo Institute of Technology, 226-8503, Japan
}

\begin{abstract}
A straight nanogroove striped pattern can be formed on the surface of $\mathrm{NiO}$ thin film by room temperature epitaxial growth in pulsed laser deposition (PLD) on a $0.2 \mathrm{~nm}$-high regularly stepped sapphire $\left(\alpha-\mathrm{Al}_{2} \mathrm{O}_{3}\right.$ single crystal) (0001) substrate and post annealing of the film. The interval and depth of each nanogroove were about 80 and $20 \mathrm{~nm}$, respectively. Wedge shaped nanogrooves were observed on the entire film $\left(\sim 10 \times 10 \mathrm{~mm}^{2}\right)$ and characterized on an atomic scale by high resolution transmission electron microscope (TEM). Using the nanogrooved $\mathrm{NiO}$ thin film or atomically stepped sapphire substrate as thermal nanoimprint mold for glass, surface nanopatterning of the silicate glass plate was successfully carried out. The straight nanogroove pattern or sub-nanometer stepped pattern were inversely transferred onto the glass surface. Growth control of transparent conducting indium tin oxide (ITO) thin films was then examined on the nanoscale step-patterned glass plates by PLD. The surface morphology of the obtained ITO thin films reflected the stepped pattern of the glass plate well. Strongly oriented film growth was achieved on the nanopatterned glass. The resistivity of ITO thin film deposited on the nanoimprinted glass was lower than that of the film on the commercial glass.

DOI: $10.2961 / \mathrm{jlmn} .2009 .03 .0011$
\end{abstract}

Keywords: Crystal growth, nanoscale surface control, nanoimprint, oxide, NiO, Transparent conductive oxide

\section{Introduction}

There has been great interest in fabricating nanostructures with a wide range of applications that include nanoelectrochemical systems, patterned nanostructures for nanoimprint, etc [1-2]. The development of nanoscale processing techniques has enabled us to fabricate novel nanostructures of scientific and applied interest. A variety of nanoscale fabrication techniques, that include have been available, e.g. electron-beam lithography, nanoimprint lithography and focused ion beam processing, are available [3-6].

Nanoimprint lithography is a promising technology for fabricating nanopatterns simply and at a low cost [4]. Thermal nanoimprint replication of nanopatterns can be performed by a single step hot pressing technique, in which the nanopatterned mold is pressed onto glassy materials, such as polymers or oxide glasses, at temperatures near their glass transition temperatures (Tg) [7-9]. Recently, we reported the nanoscale modification of silicate glass plates by applying a thermal nanoimprint technique that used selfassembled nanopattern molds of oxides, such as $\mathrm{NiO}$ or sapphire $\left(\alpha-\mathrm{Al}_{2} \mathrm{O}_{3}\right.$ single crystal) [10-11].

Nanoscale self assembly methods can also be used in construction of nanopatterns, in which nanostructures are directly built from migration of separate atoms such as nanoporous alumina and atomically stepped sapphire [1213]. The sapphire substrate used has atomically flat sur- faces with $0.2-\mathrm{nm}$ high atomic steps formed by atom migration to reduce the surface energy during thermal annealing [8]. We had previously reported fabrication of the magnetic or semiconductor nanowires, nanodots, and nanogrooves of oxides, such as $\left(\mathrm{Mn}_{0.55} \mathrm{Zn}_{0.35} \mathrm{Fe}_{0.10}\right) \mathrm{Fe}_{2} \mathrm{O}_{4}$, $\mathrm{Fe}_{3} \mathrm{O}_{4}$, and $\mathrm{NiO}$, by the self assembled decoration of atomic step edges on a sapphire substrate during film growth in pulsed laser deposition (PLD) [14-20]. $\mathrm{NiO}$ is an attractive $p$-type semiconductor with a stable wide-band-gap. The resistivity of $\mathrm{NiO}$ can be controlled by injecting lithium-ion dopant and generating hole carriers. $\mathrm{NiO}: \mathrm{Li}$ is a promising material for various applications such as high performance thermoelectric devices and cathodes of molten carbon fuel cells [21-23].

On the other hand, indium tin oxide (ITO) has been widely used as transparent electrode for displays and solar cells due to its high electric conductivity $\left(\sim 10^{-4} \Omega \mathrm{cm}\right)$ and high transmittance $(\sim 90 \%)$ in the visible region. In most optoelectronic applications, a high quality ITO thin film layer is desired to enhance the performance of a device system. Epitaxial or polycrystalline growth of ITO thin films on single crystal yttria stabilized zirconia, glass, and polymer substrate has been reported [24-27]. These studies on film growth mostly use the commercial substrates with mirror-polished or flat surfaces. There have been few studies on the crystal growth of ITO film using the substrates with artificial nanopatterned surface. The use of nanoim- 
printed glass plates for ITO thin film deposition is expected to result in a reduction of the resistivity due to homogenization of crystal nucleation sites and aligned grain growth on the regular nanopatterned glass surface.

In this paper, we present some results on the detailed formation process of nanogroove patterns formed on a $\mathrm{NiO}$ thin film surface on the atomically stepped sapphire, fabrication of nanopatterned glass using a self assembled oxide mold, and characterization of ITO thin film deposited on the nanoimprinted glass plate.

\section{Experimental}

$P$-type oxide semiconductor thin films of Li-doped $\mathrm{NiO}$ were grown on atomically stepped sapphire (0001) substrates by PLD using a $\mathrm{KrF}$ excimer laser (wave length of $248 \mathrm{~nm}$, pulse duration of $20 \mathrm{~ns}$, repetition of $5 \mathrm{~Hz}$, energy density of $3.0 \mathrm{~J} / \mathrm{cm}^{2}$ ) and a sintered target of $\mathrm{Li} 10 \mathrm{~mol} \%$ doped NiO. An atomically stepped sapphire substrate with atomically flat terrace and periodically aligned straight atomic steps was obtained by thermal annealing of a mirror polished sapphire substrate at temperatures from 1000 to $1400{ }^{\circ} \mathrm{C}$ for $3 \mathrm{~h}$ in air. $\mathrm{NiO}$ film deposition was conducted at room temperature (RT) in a $1 \times 10^{-5}$ Torr $\mathrm{O}_{2}$ atmosphere. Post annealing of an as-deposited $\mathrm{NiO}$ film was conducted in the range of 700 to $900{ }^{\circ} \mathrm{C}$ for $3 \mathrm{~h}$ in air to obtain the nanogroove structure on the $\mathrm{NiO}$ film surface.

Thermal nanoimprint of a glass plate was performed using nanogrooved $\mathrm{NiO}$ or an atomically stepped sapphire mold on a silicate glass plate. The glass transition temperature $(\mathrm{Tg})$ of the silicate glass was $521{ }^{\circ} \mathrm{C}$. The nanopatterned mold was put in contact with the surface of the glass plate and heated at $600{ }^{\circ} \mathrm{C}$ and pressed at $3 \mathrm{MPa}$ for a few minutes in vacuum.

ITO film deposition on the nanoimprinted glass substrate was carried out by PLD using a sintered target of 5 $\mathrm{wt} \% \mathrm{Sn}$-doped $\mathrm{In}_{2} \mathrm{O}_{3}$ (ITO). The film deposition was conducted at RT under a $1 \times 10^{-2}$ Torr $\mathrm{O}_{2}$ Pressure. Asdeposited film was then annealed for crystallization in a vacuum $\left(1 \times 10^{-7}\right.$ Torr $)$ at 100 to $300{ }^{\circ} \mathrm{C}$ for $3 \mathrm{~h}$.

The crystallinity of the film was examined by in situ reflection high energy electron diffraction (RHEED) and $e x$ situ X-ray diffraction (XRD). Atomic scale observation of the surface and cross section were conducted by atomic force microscopy (AFM) (SII SPI-3700) and a high resolution transmission electron microscope (HRTEM) (JEM$2100 \mathrm{~F}, \mathrm{JEOL})$. The resistivity of the films was determined by the four probe method.

\section{Results and discussion}

\subsection{Nanogroove formation in $\mathrm{NiO}$ thin film grown on the atomically stepped substrate}

Figure 1 (a) shows an AFM surface image of the stepped sapphire (0001) substrate. The substrate surface is found to have atomically smooth terraces and straight atomic steps. The terrace width was about $80 \mathrm{~nm}$ and all the step height was estimated to be $0.22 \mathrm{~nm}$, which corresponded to $1 / 6$ of the length of the c-axis of a sapphire unit cell with a corundum structure. Straight atomic steps were observed over the entire substrate surface $\left(\sim 10 \times 10 \mathrm{~mm}^{2}\right)$. Figure 1 (b) shows an AFM surface image of the NiO:Li film as-deposited. The NiO film has a smooth surface and the atomic steps were observed on the film reflecting the atomically stepped substrate surface, which indicates suppression of grain growth and thermal roughening due to the low temperature deposition. The $\mathrm{NiO}$ films were epitaxially grown on the atomically stepped sapphire substrate with a $\mathrm{NiO}(111) / /$ sapphire (0001) relationship at room temperature, as reported previously [28].

After the deposition, the epitaxial NiO:Li thin film was annealed at $700{ }^{\circ} \mathrm{C}$ for $3 \mathrm{~h}$ in air. The post annealed $\mathrm{NiO}$ film maintained the epitaxy. Figure 1 (c) shows an AFM surface image of the $\mathrm{NiO}$ thin film post annealed for $3 \mathrm{~h}$. The straight nanogroove striped pattern was clearly formed on the surface of the NiO film. The estimated depth and separation of the nanogrooves were about 20 and $80 \mathrm{~nm}$, respectively. The depth of the nanogroove was about seven times deeper than for nondoped $\mathrm{NiO}$ thin film. Impurity doping is considered to generate diffusion and reconstruction for nanogroove formation during thermal annealing. The separation of the nanogroove corresponded well with the separation of atomic steps on the sapphire substrate, as shown in Fig.1 (a). Straight nanogrooves were formed over the entire surface of the $\mathrm{NiO}$ thin film; however, nanogrooves were not formed on the $\mathrm{NiO}$ film surface grown on the mirror polished sapphire substrate. This indicates that the atomic steps of the sapphire substrate strongly affect the formation of nanogrooves on the film.

To examine the nanogroove structure and $\mathrm{NiO} /$ sapphire interface on an atomic scale, a cross sectional lattice and plan view image of TEM were taken for the annealed NiO:Li film. Figure 2 (a) shows the cross-sectional TEM image of the NiO:Li film on the sapphire substrate after annealing at $900{ }^{\circ} \mathrm{C}$. In this sample, the atomic steps were aligned parallel to the sapphire [11-20] with an average interval of about $80 \mathrm{~nm}$. The cross-sectional profile of the nanogroove was trapezoidal or wedge shaped, and the channel depth observed by TEM was consistent with the channel depth estimated by AFM. Grain boundaries normal

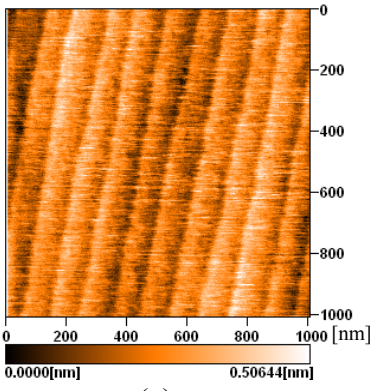

(a)

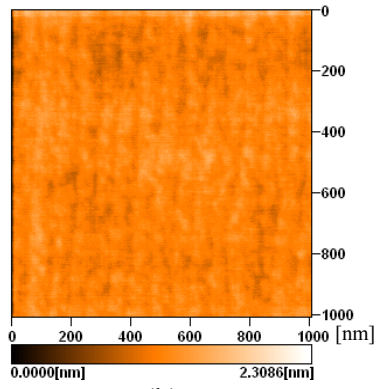

(b)

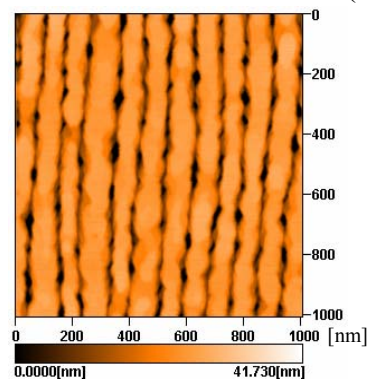

(c)

Fig. 1 AFM surface images $\left(1 \times 1 \mu \mathrm{m}^{2}\right)$ of (a) stepped sapphire substrate, (b) as-deposited NiO:Li thin film, and (c) NiO:Li thin film after annealing at $700{ }^{\circ} \mathrm{C}$. 


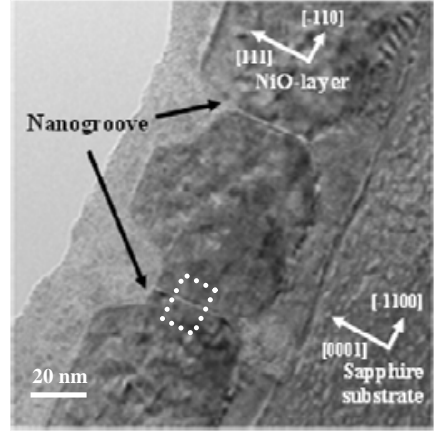

(a)

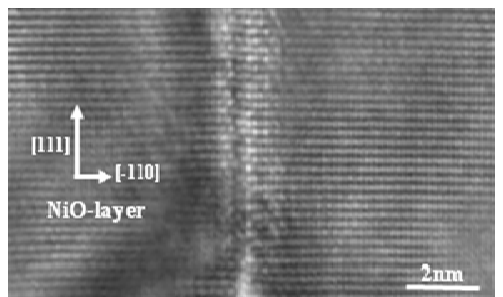

(b)

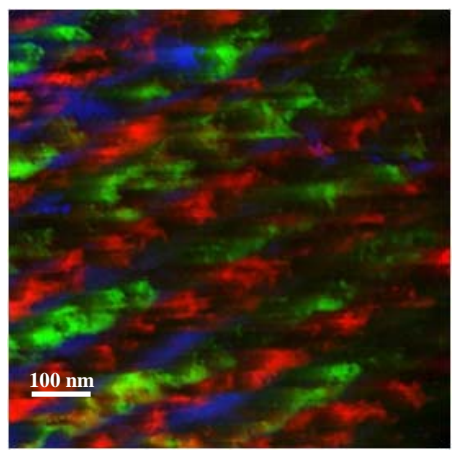

(c)

Fig. 2 TEM images of (a) cross sectional photograph, (b) magnified photogragh of dotted frame in Fig. 2 (a), (c) dark-field image in plan view of the different crystal domains (red: domain A, green: domain $\mathrm{B}$, blue: domain $\mathrm{C}$ ) observed for the NiO:Li nanopattern on sapphire substrate after annealing at $900{ }^{\circ} \mathrm{C}$.

to the substrate surface were clearly observed; these boundaries were found to connect with the bottom of the nanogroove. Figure 2 (b) shows the magnified photograph of dotted frame in Fig. (a). The displacement of the crystal lattice corresponding to $1 / 3$ of $\mathrm{NiO}$ [111] (about $0.24 \mathrm{~nm}$ ) was observed under the bottom of the nanogroove was observed in Fig. 2 (b). This indicates that the stacking sequences of $\mathrm{NiO}$ across the grain boundaries are mismatched and the displacement originating from the step bump (step height of $0.22 \mathrm{~nm}$ ) of the sapphire substrate. Figure 2 (b) shows the dark field TEM image in a plan view of the different crystal domains (red: domain A, green: domain $\mathrm{B}$, blue: domain $\mathrm{C}$ ). These domains have different in-plane orientations and domains $\mathrm{A}$ and $\mathrm{B}$ are $60^{\circ}$ rotational twins. From Fig. 2 (b), large area of each terrace is dominated by domain A or domain B and adjacent terrace has a different domain, which corresponded to the inplane sixfold symmetry of the $\mathrm{NiO}$ thin film on the sapphire substrate [28-29]. It suggests that the nanogroove formation of $\mathrm{NiO}$ film is related to the displacement of crystal lattice and the domain structure of $\mathrm{NiO}$.

\subsection{Nanopatterning of the glass surface by thermal nanoimprint with nanostructured oxide molds}

The atomically stepped sapphire substrate shown in Fig. 1 (a) and the nanogrooved $\mathrm{NiO}$ film in Fig. 1 (c) are thought to be suitable as thermal nanoimprint molds for oxide glasses, partly because of their high thermal stability and durability against oxidation and mechanical stress. Using the NiO: Li thin film with a regular nanogroove array or stepped sapphire as a nanoimprint mold, nanoimprint of the silicate glass was carried out. The step height of the sapphire mold could be controlled by changing the crystallographic surface of the sapphire substrate, as well as step bunching during high-temperature annealing, while the step separation could be controlled by changing the miscut angle of the substrate surface.

Figure 3(a) shows an AFM surface image of the nanoimprinted glass using the $\mathrm{NiO}$ mold, as shown in Fig. 1 (c) (groove depth of about $20 \mathrm{~nm}$ and separation of about 80 $\mathrm{nm}$ ). The straight nanowire pattern was transferred onto the glass surface from the nanogroove striped mold. The estimated height and separation of the nanowires on the glass surface were about 20 and $80 \mathrm{~nm}$, respectively. This corresponds well to the distance of the nanogrooves on the $\mathrm{NiO}$ mold shown in Fig. 1 (c).

Figure 3 (b) shows the surface morphology of the silicate glass nanoimprinted using the stepped sapphire mold (step height of $0.2 \mathrm{~nm}$ and step separation of about $80 \mathrm{~nm}$ ). The regular atomic steps and terraces were observed on the glass surface. The step height and separation of the nanoimprinted surface were about 0.2 and $80 \mathrm{~nm}$, respectively. It is expected that the present result can lead to morphologic control of the glass surface on an atomic scale, opening a new field in glass nanotechnology.
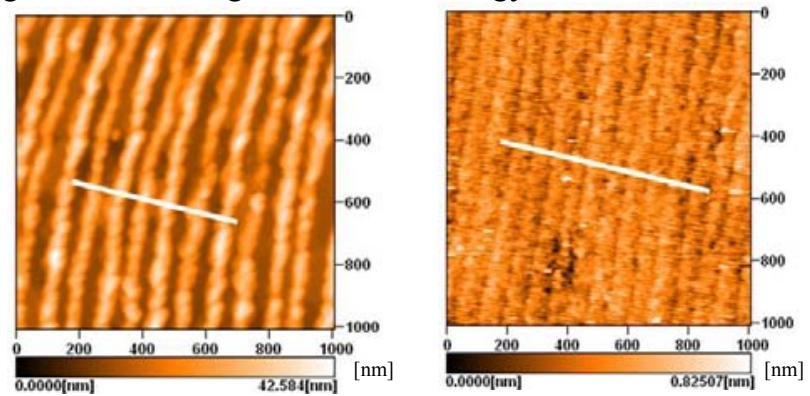

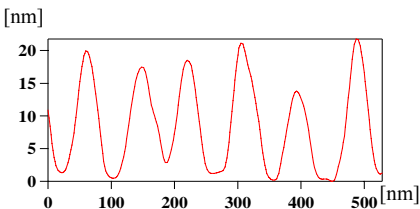

(a)

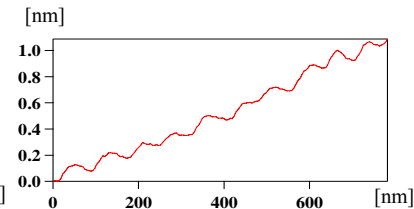

(b)
Fig. 3 AFM surface images $\left(1 \times 1 \mu \mathrm{m}^{2}\right)$ (upper) and cross sectional profiles (lower) of (a) nanoimprinted glass with $\mathrm{NiO}$ mold and (b) nanoimprinted glass with stepped sapphire mold.

\subsection{Crystal growth of ITO thin films on the nanopat- terned glass plate}

Here we examined the fabrication of ITO thin films on the nanoimprinted glass plates by PLD. Figure 4 (a) shows the AFM surface image of the nanoimprinted glass with regular steps and terraces (step height of about $2 \mathrm{~nm}$ and step separation of about $1 \mu \mathrm{m}$ ). The use of nanoimprinted glass plates for ITO thin film fabrication is expected to 


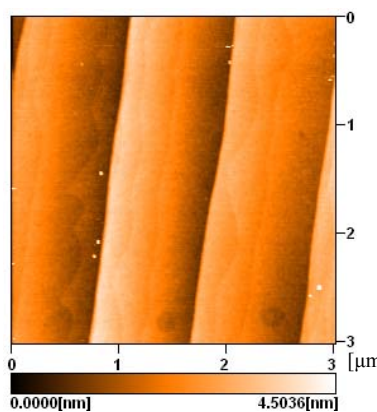

(a)

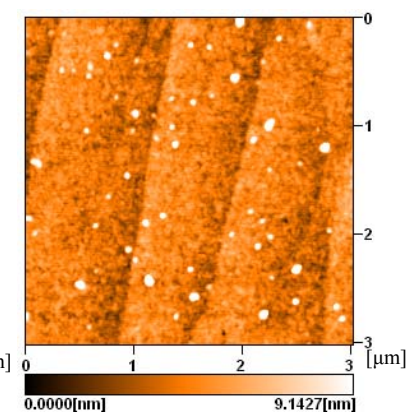

(b)
Fig. 4 AFM surface images $\left(3 \times 3 \mu \mathrm{m}^{2}\right)$ of (a) nanoimprinted glass substrate and (b) ITO thin film annealed at $300{ }^{\circ} \mathrm{C}$ after deposition on the nanoimprinted glass.

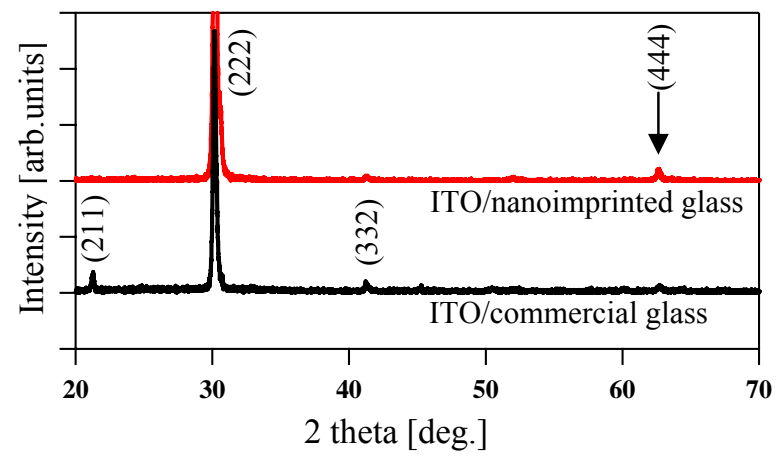

Fig.5 XRD profiles of ITO film annealed at $300{ }^{\circ} \mathrm{C}$ after deposition on the nanoimprinted glass (upper) and that on the nonpatterned commercial glass (lower).

reduce of the resistivity, probably due to the homogenization of crystal nucleation sites and aligned grain growth on the regular step patterned glass surface. The ITO thin films were deposited on the nanoimprinted glass as well as nonpatterned commercial glass. Figure 4 (b) shows the surface morphology of the ITO film (300 nm thick) annealed at $300{ }^{\circ} \mathrm{C}$ after being deposited at RT on the nanoimprinted glass. As expected, the ITO film has a step and terrace morphology, reflecting the surface morphology of the nanoimprinted glass substrate, implying that the crystal nucleation and growth of the ITO film mainly occurred with reflecting the step edge of the nanoimprinted glass.

Figure 5 shows the XRD pattern of the ITO film annealed at $300{ }^{\circ} \mathrm{C}$ after being deposited at RT on the nanoimprinted and that on the non-patterned commercial glass. The ITO film found to exhibit strong (111) oriented polycrystalline growth on the nanoimprinted glass plate. We also found that the orientation of the ITO film fabricated on the nanoimprinted glass was stronger than that on the commercial glass plate. The preferential orientation of ITO film growth on the nanoimprinted glass plate is probably due to homogenization of crystal nucleation sites on the edge of regularly steps and growth on the flat terrace surface of the nanoinprinted glass.

Table 1 exhibits the resistivity at RT for the ITO film annealed at $300{ }^{\circ} \mathrm{C}$ after deposition at RT on the commercial and nanoimprinted glass plates. A low resistivity of 4.6 $\times 10^{-4} \Omega \mathrm{cm}$, was obtained for the ITO thin film deposited on the nanoimprinted glass after annealing at $300{ }^{\circ} \mathrm{C}$. The resistivity of the ITO film deposited on the nanoimprinted glass is lower than that of the film on the commercial glasses, as shown in Table 1. We think the stronger crystalline orientation of the film under the appropriate conditions is expected to reduce carrier scattering, i.e., an increase in carrier mobility for the ITO film on the nanoimprinted glass. Further experiments should clarify the relationship between the ITO film crystalline orientation and the resistivity of the film.

Table 1 Resistivity at RT of ITO films annealed at $300^{\circ} \mathrm{C}$ after deposition.

\begin{tabular}{lc}
\hline Substrate & Resistivity $\left(\times 10^{-4} \Omega \mathrm{cm}\right)$ \\
\hline Commercial glass & 5.5 \\
Nanoimprinted glass & 4.6 \\
\hline
\end{tabular}

\section{Conclusion}

A straight nanogroove pattern could be formed on the surface of epitaxial $\mathrm{NiO}$ thin film on a $0.2 \mathrm{~nm}$ high regularly stepped sapphire substrate. The separation of the nanogroove corresponded to the separation of atomic steps on the sapphire substrate. The nanogroove formation of $\mathrm{NiO}$ seems to be related to the displacement of the crystal latticeand the domain structure of $\mathrm{NiO}$. Using the nanogrooved $\mathrm{NiO}$ thin film of the atomically stepped sapphire substrate as a thermal nanoimprint mold for glass, straight nanowire pattern or sub-nanometer stepped pattern was transferred onto the glass surface. The ITO thin films were deposited on the nanoimprinted glass with regular steps and terrace. Preferential crystalline orientation of the ITO thin films was achieved and the resistivity was lower than that of the film on the commercial glass.

\section{Acknowledgments}

This work was supported in part by the Ministry of Education, Culture, Sports, Science and Technology of Japan, the National Institute of Advanced Industrial Science and Technology of Japan, the New Energy and Industrial Technology Development Organization of Japan, and the Regional Innovation Creation R\&D Program from the Ministry of Economy, Trade, and Industry of Japan.

\section{References}

[1] D. F. Wang, T. Ono and M. Esashi: Nanotechnology, $15,(2004) 1851$

[2] S. Y. Chou, P. R. Krauss and P. J. Renstrom: Science, 272, (1996) 85

[3] A. N. Broers: J. Electrochem. Soc., 128, (1981) 166

[4] S. Y. Chou, P. R. Krauss and P. J. Renstrom: Appl. Phys. Lett., 67, (1995) 3114

[5] S. Matsui, K. Mori, K. Saigo, T. Shiokawa, K. Tadoya and S. Namba: J. Vac. Sci. Technol. B, 4, (1986) 845

[6] B. Klehn and U. Kunze: J. Appl. Phys., 85, (1999) 3897

[7] B. Heidari, I. Mximov, E. L. Sarwe and L. Montelius: J. Vac. Sci. Technol. B, 17, (1999) 2961

[8] T. Morita, K. Watanabe, R. Komatani, K. Kanda, Y. Haruyama, T. Kaito, J. Fujita, M. Ishida, Y. Ochiai, T. 
Tajima and S. Matsui: Jpn. J. Appl. Phys., 42, (2003) 3874

[9] Y. Hirai, K. Hunakugi, T. Yamaguchi, K. Yao, S. Kitagawa and Y. Tanaka: Microelectron. Eng., 67-68, (2003) 237

[10] S. Akiba, W. Hara, T. Watanabe, A. Matsuda, M. Kasahara and M. Yoshimoto: Appl. Surf. Sci., 253, (2007) 4512

[11] Y. Akita, T. Watanabe, W. Hara, A. Matsuda and M. Yoshimoto: Jpn. J. Appl. Phys., 46, (2007) L342

[12]H. Masuda, H. Yamada, M. Satoh, H. Asou, M, Nakano and T. Tamamura: Appl. Phys. Lett., 71, (1997) 2770

[13] M. Yoshimoto, T. Maeda, T. Ohnishi, H. Koinuma, O. Ishiyama, M. Shinohara, M. Kubo, R. Miura and A. Miyamoto: Appl. Phys. Lett., 67, (1995) 2615

[14] M. Yoshimoto, A. Sasaki and S. Akiba: Sci. Technol. Adv. Mater., 5, (2004) 527

[15] M. Takakura, T. Miyahara, J. Tashiro, A. Sasaki, M. Furusawa and M. Yoshimoto: Mater. Res. Soc. Symp. Proc., 648, (2001) 6.5.1

[16] M. Yoshimoto, K.Mizuno and T. Miyahara: Mater. Res. Soc. Symp. Proc., 587, (2000) O3.1.1

[17] A. Sasaki, S. Akiba, A, Matsuda, W. Hara, S. Sato and M. Yoshimoto: Jpn. J. Appl. Phys., Part 1, 44, (2005) 256

[18] O. Sakata, A. Kitano, W. Yashiro, K. Sakamoto, K. Miki, A. Matsuda, W. Hara, S. Akiba and M. Yoshi- moto: Mater. Res. Soc. Symp. Proc., 840, (2005) Q6.4.1

[19] O. Sakata, M. Takata, H. Suematsu, A. Matsuda, S. Akiba, A. Sasaki and M. Yoshimoto: Appl. Phys. Lett., 84, (2004) 4239

[20] S. Akiba, A. Matsuda, H. Isa, M. Kasahara, S. Sato, T. Watanabe, W. Hara and M. Yoshimoto: Nanotechnology, 17, (2006) 4053

[21] W. Shin and N. Murayama: Mater. Lett., 45, (2000) 302

[22] R. C. Makkus, K. Hemmes and J. H. W. de Wit: J. Electrochem. Soc., 141, (1994) 3429

[23] S. Kim, S. P. Yoon, J. Han, S. W. Nam, T. H. Lin, I. Oh and S. Hong: Electrochem. Acta, 49, (2004) 3081

[24] H. Ohta, M. Orita and M. Hirano: Appl. Phys. Lett., 76, (2000) 2740

[25] A. Suzuki, T. Matsushita, T. Aoki, A. Mori and M. Okuda: Thin solid films, 411, (2002) 23

[26] F. O. Adurodija, H. Izumi, T. Ishihara, H. Yoshioka, M. Motoyama, K. Murai: Jpn. J. Appl. Phys., 39, (2000) L377

[27] C. Guillen and J. Herrero: Thin solid films, 480, (2005) 129

[28] Y. Kakei, S. Nakao, K. Satoh, and T. Kusaka: J. Cryst. Growth, 237-239, (2002) 591

[29] O. Sakata, M. S. Yi, A. Matsuda, J. Liu, S. Sato, S. Akiba, A. Sasaki and M. Yoshimoto: Appl. Surf. Sci., $221,(2004) 450$ 